2. Early left hemispheric lesions may induce reorganisation to the right - in homotopic areas.

3. This happens on the expense of right hemispheric functions.

4. Language quality in reorganised language is not impaied for everyday language, but there are differences in complex linguistic aspects, which supports the idea of a genetic predisposition.

5. The time frame for reorganisation is not very clear, but there is no evidence for successful reorganisation after preschool age.

This work is supported by the German Research Council (DFG) and the University of Tübingen.

\section{The Child}

\section{IS-022 CRITICAL CARE FOR THE CHILDREN OF THE WORLD}

N Kissoon. Global Child Health, BC Children's Hospital and University of British Columbia, Vancouver, Canada

\subsection{6/archdischild-2014-307384.22}

The burden of sepsis in the developing world Early childhood and neonatal sepsis has become a global public health concern. Nearly seven million children die each year before reaching their fifth birthday and around 50\% of these deaths are attributable to sepsis and possible serious infections.

Studies have demonstrated the effectiveness of community interventions in reducing neonatal deaths due to sepsis and other severe diseases. But in resource poor settings, the lack of access to health facilities, acute shortage of a trained health workforce and unsatisfactory diagnosis and treatment in the facilities have been identified as some of the major contributors to high sepsis related mortality. Sepsis should be recognised early and treated aggressively. Therefore the quality of care at health facilities needs to be ensured to provide comprehensive management to all sepsis cases in order to prevent or decrease the onset of fatal consequences like septic shock and vital organ failure. A systematic review of thirteen studies in developing country context reported that the post discharge mortality rate may vary between $1 \%$ and $18 \%$. The burden of morbidity and mortality of post discharged sepsis cases needs to be further evaluated in developing country context.

As representatives of the Global Sepsis Alliance (http://globalsepsisalliance.com/) we have had fruitful discussions with the WHO and the Global Burden for Disease experts who are eager to highlight the burden posed by sepsis. You can make a difference. Joining the World Sepsis Day initiative is a welcome start (http://www.world-sepsis-day.org/).

\section{The Gut}

\section{IS-023 CLINICAL ASPECTS OF NEONATAL CHOLESTASIS}

B Fischler. Department of Pediatrics, Karolinska University Hospital CLINTEC, Stockholm, Sweden

\subsection{6/archdischild-2014-307384.23}

Neonatal cholestasis most often presents as prolonged jaundice, beyond 2 weeks of age. Such babies should always be investigated for conjugated hyperbilirubinemia and if this is detected further work-up for cholestasis is mandatory. Simultaneously, prothrombin time must be checked and pathologic levels need to be treated with intravenous vitamin $\mathrm{K}$, to avoid detrimental bleedings. Neonatal cholestasis occurs in approximately 1 in 2500 term infants, the most common underlying diseases being biliary atresia (BA), viral infections, $a_{1}$-antitrypsin deficiency and other genetic cholestatic disorders.

Patients with BA most commonly present with jaundice and pale stools. Population based screening of all infants, by the use of stool colour cards sent to the parents can speed up the detection of cases with BA and thereby improve the success rate of the surgical procedure.

Once cholestasis is established, there is a need for a relatively rapid series of investigations. The reason for this "semiacute" management is to define certain situations where early treatment is lifesaving, such as septicemia, galactosemia or hypocortisolism, as well as to ensure timely surgical management in BA patients. A stepwise approach of investigations is suggested, where percutaneous liver biopsy is of value to select patients with suspected biliary atresia for laparotomy.

All patients, regardless of aetiology, should receive fat soluble vitamins as long as they are cholestatic. Although widely used, the effect of ursodeoxycholic acid is less well proven. However, it seems to be beneficiary in subsets of patient groups and the side effects are very minor.

\section{The Heart}

\section{IS-024 FETAL CARDIAC INTERVENTIONS}

${ }^{1} \mathrm{G}$ Tulzer, ${ }^{2} \mathrm{~W}$ Arzt. ${ }^{1}$ Department of Pediatric Cardiology, Children's Heart Center Linz, Linz, Austria; ${ }^{2}$ Institute of Prenatal Medicine, Children's Heart Center Linz, Linz, Austria

\subsection{6/archdischild-2014-307384.24}

Fetal cardiac interventions are currently performed for critical aortic stenosis (CAS), pulmonary atresia intact septum (PA/IVS) and closed atrial septum in hypoplastic left heart syndrome (HLHS). The rational is to change fetal hemodynamics, prevent secondary damage and improve long term outcome at an acceptable risk for mother and fetus.

In fetal CAS the left ventricle (LV) dilates, shortening decreases and endocardial fibroelastosis develops. The most important issue is to identify those patients, who will benefit from this procedure.

Intervention has been shown to improve fetal hemodynamics. The risk of intrauterine death is around 12\%. A biventricular outcome has been reported in $1 / 3$ to $2 / 3$ of these fetuses.

At the Children's Heart Centre Linz, 57 procedures were performed in 50 fetuses, with a success rate of $83 \%$ and a biventricular outcome after a median follow-up period of 43 months of $56 \%$.

Intervention in fetal PAIVS is technically more challenging. In our centre 12 attempts have been performed in 10 fetuses all with suprasystemic RV pressures. There was no serious complication so far. Successful intervention (in 8 fetuses) resulted in better RV filling and continuous, but slower than normal growth of tricuspid valve and RV. After 1 year, 4/5 newborns are biventricular and well, 1 child received a Glenn shunt. One 6 months old patient still has a Blalock Taussig shunt, 2 fetuses are still in-utero.

Intact atrial septum in fetal HLHS has to be treated with transseptal stent placement to prevent re-occlusion. Results however are still poor (Own experience with 5 procedures).

\section{IS-025 FUNCTIONAL ECHOCARDIOGRAPHY: CORE COMPETENCY FOR THE NEONATOLOGIST?}

N Evans. Newborn Care, RPA Hospital and University of Sydney, Sydney, Australia

10.1136/archdischild-2014-307384.25 